Military Technical College

Kobry El-Kobbah

Cairo, Egypt

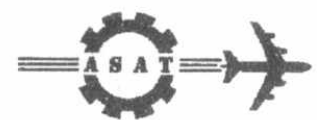

$10^{\text {th }}$ International Conference

On Aerospace Sciences\&

Aviation Technology

\title{
Design and Implementation of an ECG Transmitter- Receiver through Telephone Networks
}

\author{
A.S. Ragab and Mahmoud. E. Gadallah
}

Armed forces

\begin{abstract}
This paper introduces a design as well as an implementation for a system capable for extracting the electrocardiograph (ECG) signal from a patient and transmitting it over telephone networks to a remote site where it is received and displayed on an oscilloscope and stored in a PC computer. The introduced system in this work has been tested via real experimentation on a public telephone network.
\end{abstract}

\section{KEY WORDS}

ECG, ECG acquisition, Biomedical transmitters implementation and Biomedical receivers implementation.

\section{I: INTRODUCTION}

It has been proved medically that in some cases of heart diseases, any delay in the diagnosis and fast advice by the physician may cost the patient his life [1]. Also, some of the heart pains may appear at any time and anywhere. So, it is important to enable the patient to send his ECG signals immediately to the medical care site. These remarks have been the motivations behind the work introduced in this paper.

The medical systems that can perform the transmission of the ECG signals over the telephone network are few. One of them produced by Reynolds which is called "Cardio call", is designed such that has to store the ECG signals first for a limited time and then the transmitter has to be put on the microphone of the telephone handset for transmission. This method of operation causes some problems such as:

1. Any mis-positioning of the transmitter on the microphone of the handset or any nonintentionally movement results in additional noise and destruction of the ECG signals

2. The environmental noise in the transmission site corrupts the ECG signals.

These problems have been overcome in the system introduced in this work 
The paper is organized as follows: the proposed system is described in section 2 . In section 3 , the evaluation tests that have been performed are introduced. The drawn conclusions from this work are given in section 4

\section{DESCRIPTION OF THE PROPOSED SYSTEM}

The proposed system in this work has to be perform the following functions:

1. ECG signal extraction in the patient site.

2. Transmitting the extracted ECG signal over the telephone network.

3. Receiving the ECG signal in the medical center site.

4. Displaying the received ECG signal on an oscilloscope and computer with the possibility of storing it for further processing

Thus, the introduced system consists of two units, as shown in Figure 1, namely the patient unit and medical center unit.

\subsection{The Patient Unit}

The patient unit comprises two modules in cascade in the same case. The first module is the ECG extractor that is followed by the ECG transmitter module as shown in Figure 2.

\subsubsection{The ECG Extraction Module}

The ECG extraction module is the part which is used to generate lead I, lead II and lead III signals of the ECG using the output of four electrodes attached to the left arm (LA), right arm (RA), left leg (LL) and the right leg (RL) of the patient. Where leads I, II and III signals are the voltage difference between LA and RA, LA and LL, and RA and LL, respectively. The $\mathrm{RL}$ voltage is used as reference voltage. The output of this stage is amplified enough to drive the reset stages and with as minimum interfered noise as possible. Thus this module consists of the following stages:

(i) A buffer stage to avoid the loading effect of the extraction circuit to the electrodes.

(ii) A differential multiplexer that is used to select one lead of the three leads. It is controlled by a mechanical switch.

(iii) A Difference amplifier to amplify the voltage difference between any two-electrode outputs that are selected by the multiplexer.

(iv) A low-pass filter with cut-off frequency $40 \mathrm{HZ}$ to remove any corrupting signals or noise [2]

(v) An inverting amplifier stage with gain 50 is used to match the extracted signal amplitude with the standard ECG signal amplitude.

\subsubsection{The ECG Transmitter Module}

Since the patient unit should be portable to let the patient carrying it easily from place to place, then its size must be minimized. For this reason, analog transmission technique is 
preferred over digital transmission technique to avoid the necessity of using digitization circuits and modems.

The ultimate aim of the ECG transmission module is to transmit the ECG signals of band width $0.1-40 \mathrm{~Hz}[2,4]$ over public telephone network lines of band width $0.3-3.4 \mathrm{kHz}[5]$, Thus the main stage of this module is a modulator to make the transmission possible. The frequency modulation (FM) scheme is selected to avoid the problems of amplitude attenuation and addition noise of the telephone line. The frequency modulation is performed using voltage-controlled oscillator (VCO). The extracted ECG signal amplitude controls the output frequency of this VCO. The output of this stage is frequency-modulated signal with center frequency $1.6 \mathrm{kHz}$ and frequency band of $800-2400 \mathrm{~Hz}$. The bandwidth is determined according to the circuit parameters of the selected $\mathrm{VCO}[6]$.

To avoid the effect of negative amplitudes on the VCO a level shifter circuit is introduced before its circuit to ensure that the VCO input is always positive. The final stage of the ECG transmission module is an audio transformer, which is used to couple the VCO output signal to the telephone line

The transmission unit must be battery supplied to avoid the possible dangerous of using the high voltages. This minimizes the use of patient protection elements in the circuit. Fig. 3 shows the circuit diagram of the ECG patient unit.

\subsection{The Medical Center Unit}

As shown in Fig. 4, this unit consists of the receiving subunit and displaying and recording station.

\subsubsection{The Receiving Unit}

The function of the receiving unit is to detect the ECG signal from the received FM signal on the telephone line. Fig. 5 shows the schematic diagram of this unit. The circuit starts with an audio transformer to couple the FM signal from the telephone line to the receiver. A frequency demodulator is used which is the same as the FM modulator but with different configuration. To compensate the line disturbance and attenuation, the received signal is amplified with gain and filtered using a law-pass filter of $40 \mathrm{~Hz}$ bandwidth.

\subsubsection{The Displaying And Recording Station}

The function of this station is to display the received ECG signal on line and store the desired parts of the displayed signal in the database assigned to the patient. This station consists of an digital oscilloscope to view the received ECG signal. This oscilloscope is interfaced with a computer. When the medical staff who takes care of the case under consideration wants to store some part of the displayed ECG signal, they can do that using the acquisition software easily. Further more he (she) can print the selected parts as a hardcopy. Fig. 6 shows two examples of the extracted ECG signal at medical care site. 


\section{Conclusion}

The work reported in this research has shown that, the idea of transmitting the ECG signals through a telephone network is feasible. In this work great attention has been paid to reduce the effect of both the noise and the attention due to the telephone network. In designing the system introduced in this work, three main points have been considered:

(a) Reducing of the size of both the transmitter and the receiver units.

(b) Ease of use at both patient site and the hospital site

(c) Minimizing of the effect of the telephone network on the ECG signal.

These considerations are important to achieve the portability of the transmitter and its easy use by the patient himself (herself). The third consideration mentioned above is very important for correct diagnosis of the patient case.

The system introduced in this research has many advantages of the similar systems in the market. Among these advantages the following:

(a) Online transmission of the ECG signal as far as the electrodes are connected the patient. The system available in the market allow the patient to store fixed interval of $20 \mathrm{~min}$ max distributed on small intervals (1-2 min), then the transmission can be accomplished.

(b) The system introduced on this work, connect the ECG signal directly to the line but the similar systems, the patient has to put the transmitter on the microphone of the telephone handset. This is an important advantage to prevent the environmental noise from corrupting and interfering with the transmitted signal.

\section{References}

[1] Jon D. Cooksey, Marvin Dunn, Edward Massie, "Clinical vector cardiography and electrocardiography" 2nd edition, Year Book Medical publisher inc., 1977.

[2] C. Li, Zheng, and C. Tai, "Detection of ECG characteristics points using Wavelet transformation", IEEE Trans. On Biomedical Eng. Vol.42, no 1, pp.21-28, 1995.

[3] K Mayyas and B. El-Asir, "Discrete Wavelet Analysis of heart Sounds Using Filter Banks" Int. Conf. On Electronics, Circuits and systems of the IEEE, Cairo, pp.10441048,1997

[4] G. Webster, Editor, "Medical Instrumentation Application and Design" Third Ed., 1998.

[5] Understanding Telephone Electronics, 3rd edition, Stephen J. Bigelow, Sams, 1991

[6] Data Handbook, HE4000B Logic Family CMOS, PHILIPS, 1990. 


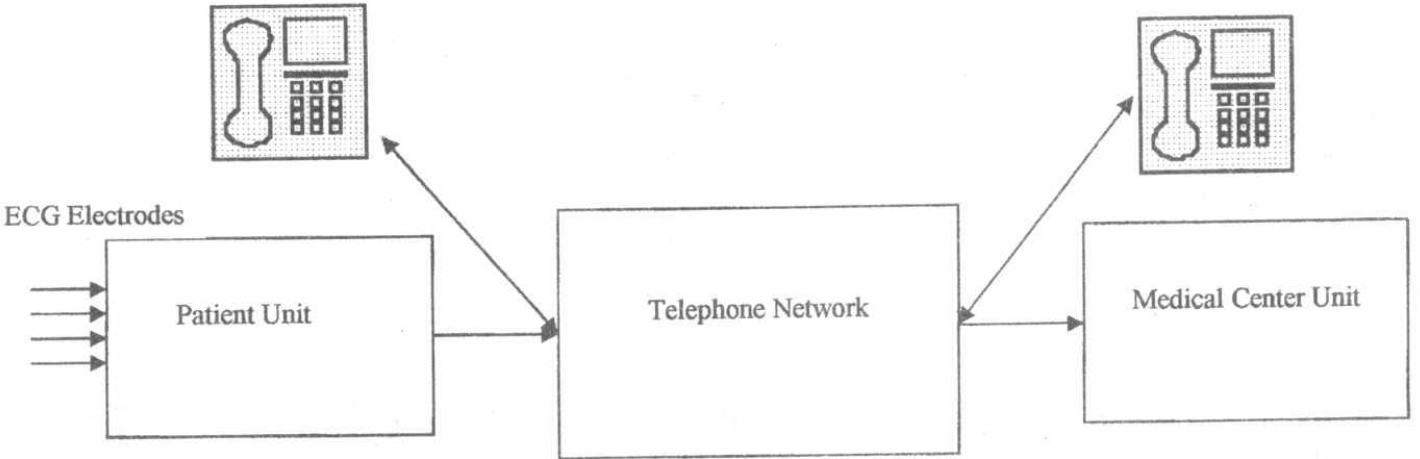

Fig. 1 Block diagram of the whole system

Telephone Set

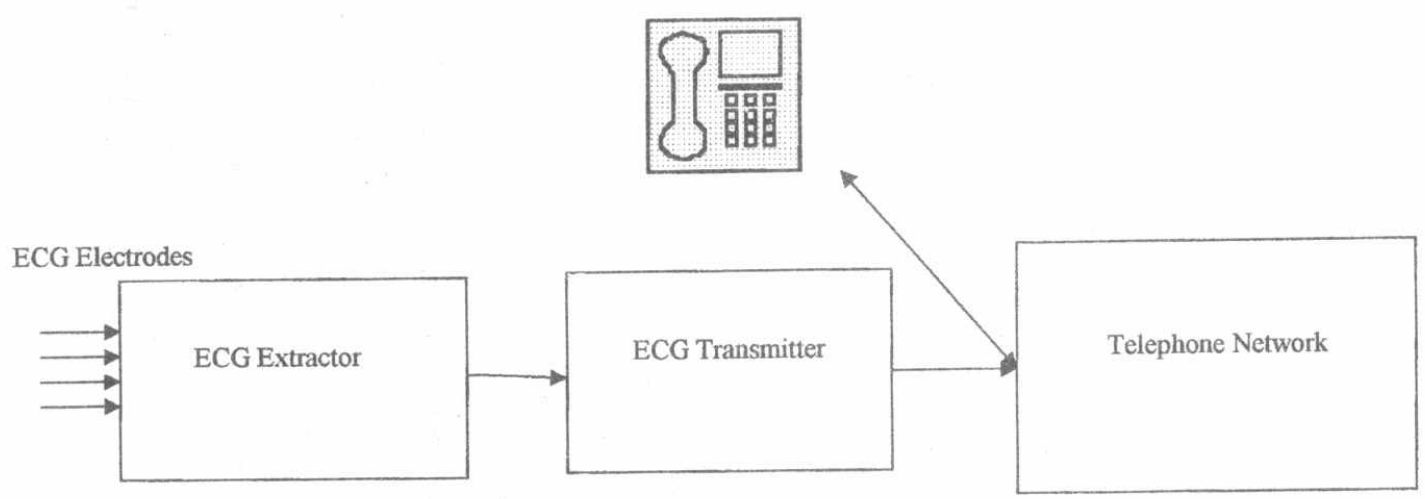

Fig. 2 Block diagram of patient unit 


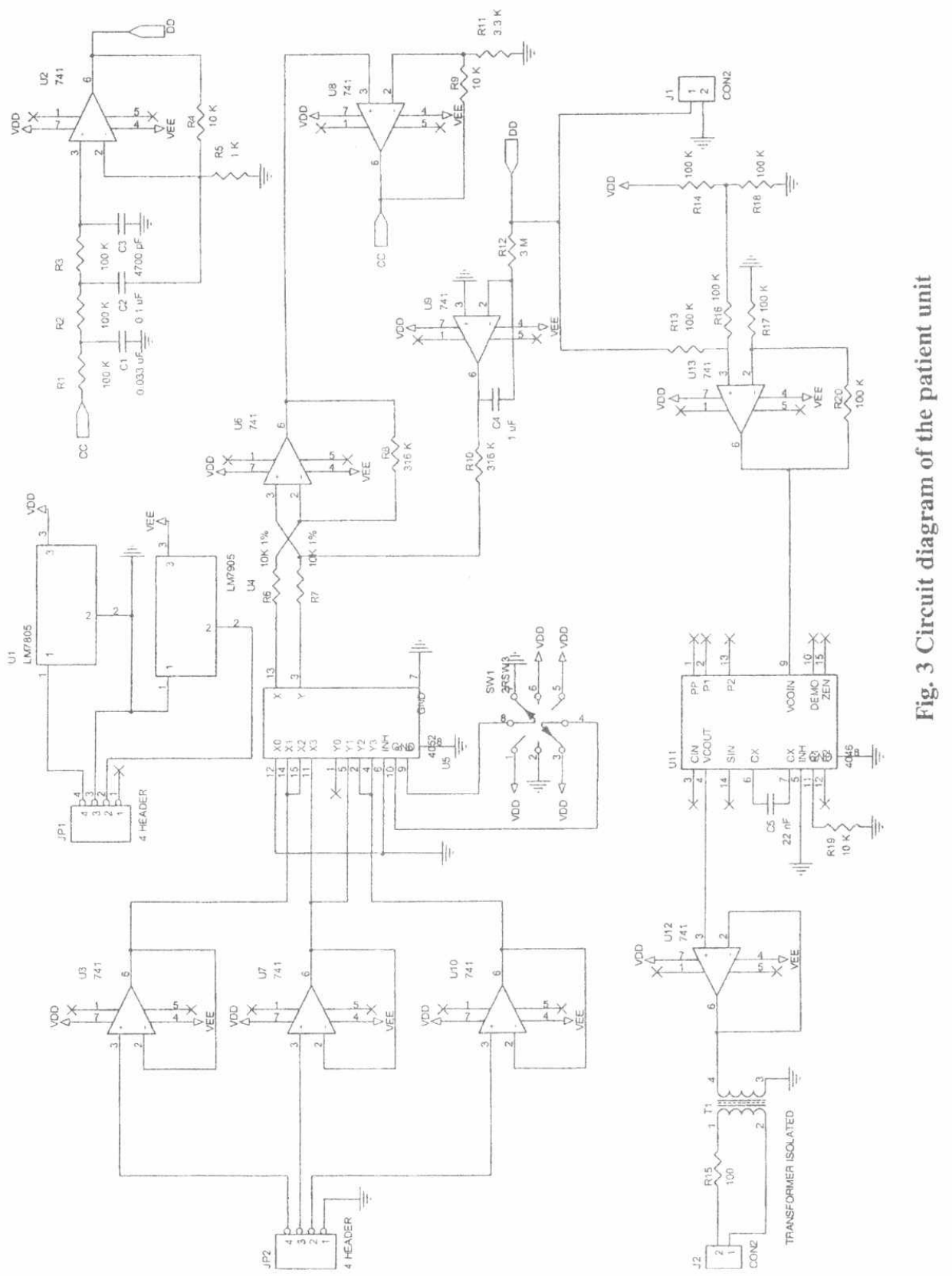




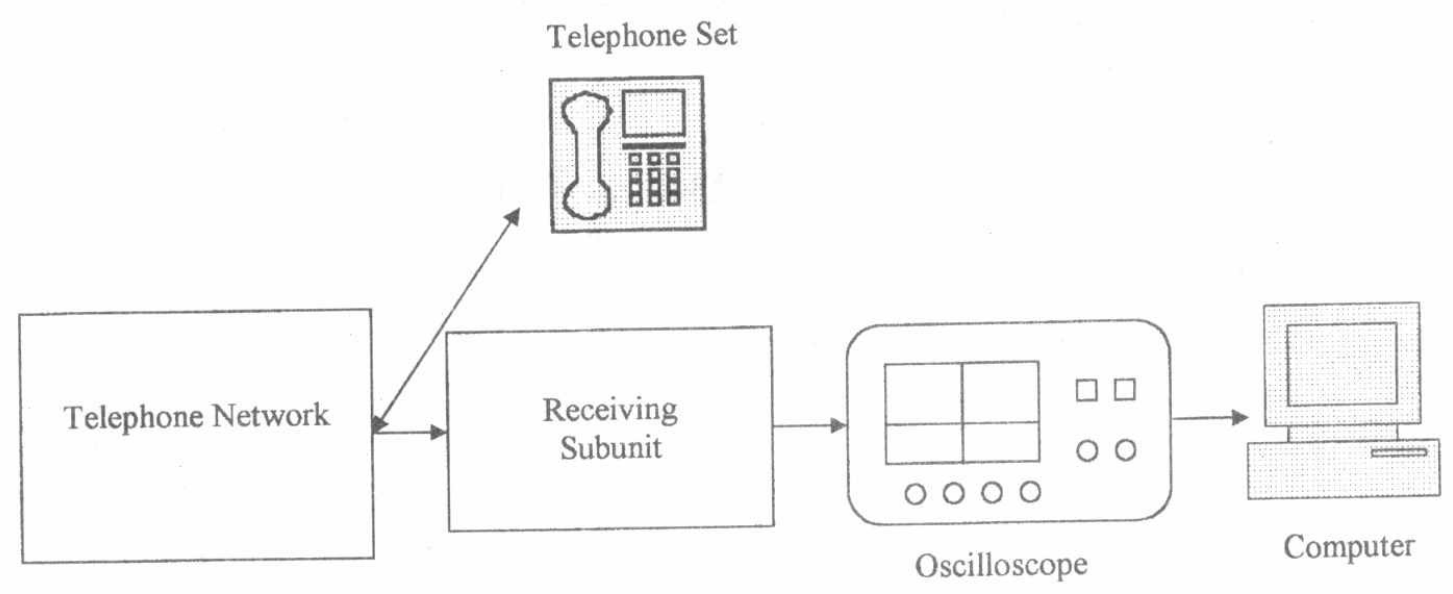

Fig. 4 Block diagram of the medical center unit

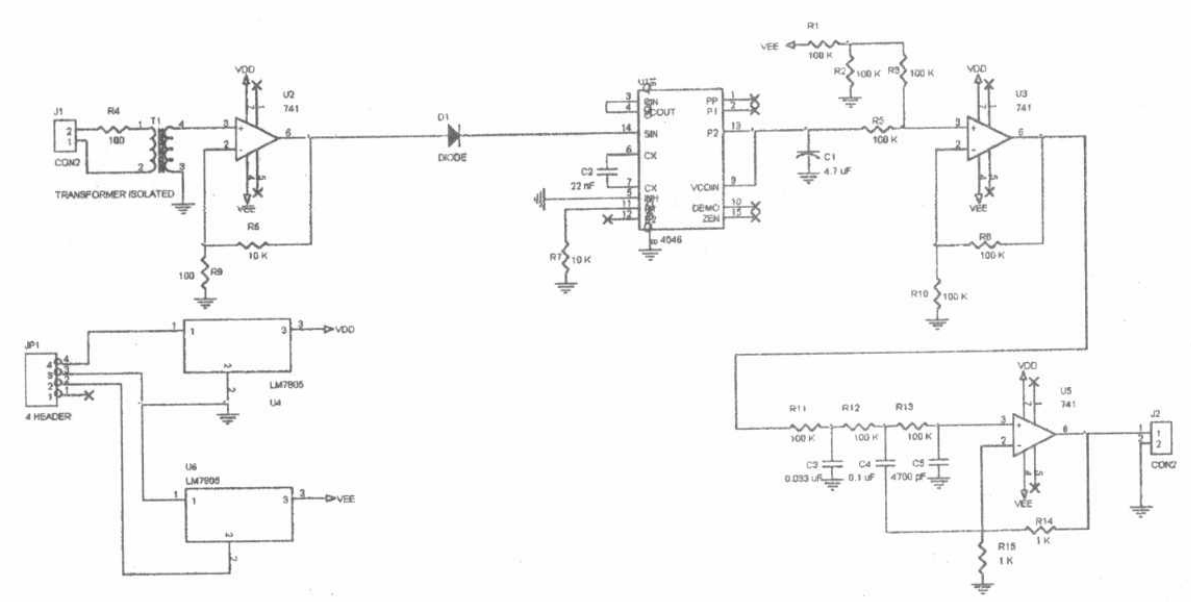

Fig. 5 Circuit diagram of the receiving subunit 


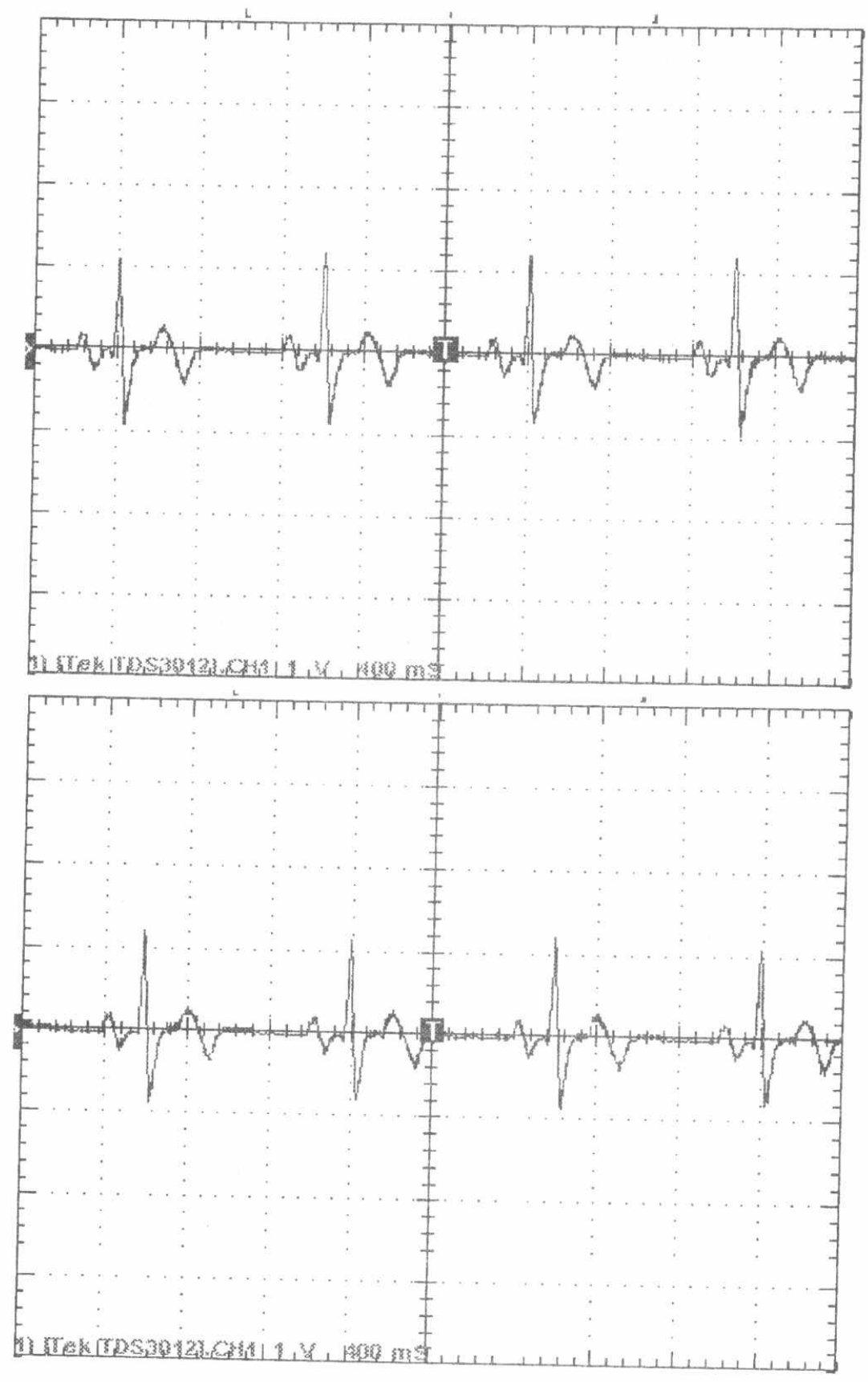

Fig. 6 Two examples of the extracted ECG Signals 